\title{
STATISTICAL ESTIMATION OF THE MAXIMUM RESPONSE OF STRUCTURES SUBJECTED TO EARTHQUAKE MOTION
}

\author{
Yoshikazu Yamada* \\ Hirokazu Takemiya**
}

\begin{abstract}
In this paper, the authors derived analytically the formula finding the absolute maximum response of structures due to earthquake motions from their respective maximum acceleration. The computation results for a given structure by this formula accompanied by the assumptions of input power spectral density gave a good coincidence within a narrow band estimation with its precise response obtained by the direct-integration of the governing equation with strongearthquake records as an input.
\end{abstract}

\section{INTRODUCTION}

Earthquake-resistant design of civil engineering structures with a long period of vibration has been based on their response analysis using certain strongearthquake records or their average response spectrum. However, these methods still have some shortcomings: in the former, due to the individual earthquake characteristics, and in the latter, due to the averaging process. As a new approach, a stochastic process, characterized by spectral or correlation method of analysis, has been applied to this field of study by many researchers ${ }^{12}{ }^{\sim 11}$. As a result, the expected mean value and moments of random response have been obtained. However, it is the maximum value that has the most vital role in structural design, and thus an attempt is made herein to derive the formula finding the maximum deformation response of structures when subjected to earthquake motion.

There are two methods of approach available to find the maximum value of random variables after their statistical processing: namely

(1) threshold-crossing method

(2) peak-distribution method

The general concept on (1) and (2) was formulated by S. O. Rice ${ }^{12}$ ) and D. Middleton $^{13)}$. Furthermore S. O. Rice showed that these two methods yielded almost the same result near the maximum in the case of stationary Gaussian white noise passed by a low-pass filter or a band-pass filter. For this reason, the computation presented herein is performed by using method (2) after the spectral analysis of random variables.

This method assumes the input power spectral density of earthquake motion.

* Dr. Eng., Professor, Dept. of Civil Eng., Kyoto University.

** M.S. (Civil), Assistant, Dept. of Transportation Eng., Kyoto University. 
Tajimi, for the first time, proposed to use as a substitute for this the absolute acceleration response of a one-degree-of-freedom system to a stationary Gaussian white noise ${ }^{1)}$. But his proposal has a discrepancy in the lower frequency range near zero which severely affects the response of structures with a long vibration period. Therefore, its improved form was adopted by H. Sato ${ }^{14)}$. In this paper the authors, taking into account the multi-predominant frequencies of earthquake motion, simulate the above spectral density of the velocity response one of an idealized foundation layer with a parallel arrangement of one-degree-of-freedom systems as an input of a stationary Gaussian white noise.

Since the intensity of earthquake motions is generally measured by their respective maximum acceleration, it is most significant to predict the corresponding maximum response of structures from this value. Tajimi, assuming that the ratios of maximum to standard deviation of both the excitation and response are equal, constructed the response amplification factors of deformation, velocity and acceleration for a structure whose natural period is shorter than twice the supposed vibration period of the foundation layer ${ }^{1)}$. In a third case though, $\mathrm{H}$. Sato corrected this by considering the extreme probability density both for the excitation and response ${ }^{14)}$. However, in a case of structures with a long vibration period, it is the deformation response that determines their design parameters. Y. Nakao and N. Sasaki, considering the joint probability density of the above ratios, deduced a formula concerning the maximum deformation response of structures to the maximum input acceleration ${ }^{15}$, but it still has a broad band estimation. Herein, the authors took the following approach for this investigation. The relationships between maximum and standard deviation, both of earthquake motions and response of one-degree-of-freedom systems, were first studied, and then, by combining these with the results from their spectral analyses, their precise response amplification was determined with a narrow band estimation. It is the function of (1) the response amplification in a stochastic sense of a concerned system, (2) its vibration period and the damping effect, and (3) the duration of the earthquake motion. This discovery makes it feasible to estimate properly the response of structures due to earthquake motions even in the case of a multi-degree-of-freedom system, as shown in the example.

\section{STATISTICAL ESTIMATION OF THE MAXIMUM RANDOM VARIABLE}

In this paper, as was mentioned in the Introduction, the absolute maximum value of random variables is found from the peak distribution. The related basic theory was reviewed by Y. K. Lin $^{16}$ in the case of a stationary stochastic process. The authors have tried further to extend it in a form applicable to a nonstationary stochastic process.

D. Middleton expressed by the following equation the expected number of peaks above the specified level during the interval of $\left(t_{1}, t_{2}\right)$, provided that the random variable $x$ is differentiable with respect to time up to the second order ${ }^{13}$.

$$
N\left(\xi, t_{1}, t_{2}\right)=\int_{t_{1}}^{t_{2}}|\ddot{x}(t)| \delta[\dot{x}(t)] l[x(t)-\xi] d t
$$

where $\delta[\cdot]$ signifies the Dirac delta function and $l[\cdot]$ the Heaviside step function, 
respectively. By the use of the joint probability density function of the involved variables in Eq. (2.1), the expected number of peaks above $\xi$ during the interval $\left(0, t_{d}\right)$ is obtained as

$$
E\left[\dot{N}\left(\xi, t_{d}\right)\right]=-\int_{0}^{t_{d}} d t \int_{\xi}^{\infty} \int_{-\infty}^{0} \ddot{x}[p(x, \dot{x}, \ddot{x} ; t)] d \ddot{x} \ddot{x} d x
$$

where $p(x, \dot{x}, \ddot{x} ; t)$, the probability density of $x(t), \dot{x}(t)$, and $\ddot{x}(t)$ at time $t$, is assumed to be subjected to the Gaussian distribution and then the corresponding at $\dot{x}=0$ is expressed in the form as

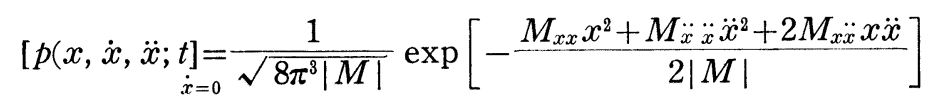

where

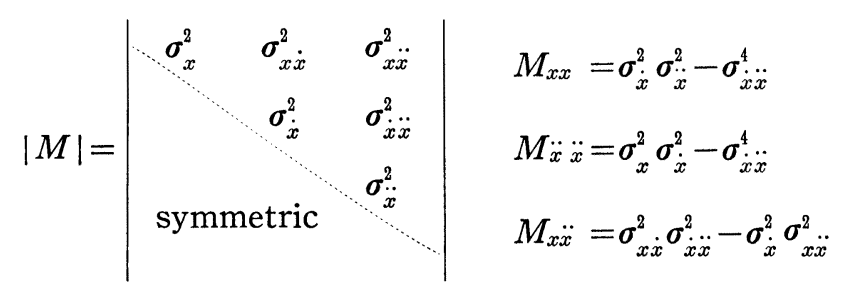

The notations $\sigma_{x}^{2}, \sigma_{\dot{x}}^{2}, \sigma_{\ddot{x}}^{2} ; \sigma_{x \dot{x}}^{2}, \sigma_{x \ddot{x}}^{2}, \sigma_{\ddot{x} \ddot{x}}^{2}$ are the variances of random variables $x(t)$, $\dot{x}(t)$ and $\ddot{x}(t)$, and their covariances, respectively, which are obtained through stochastic processing. The number of peaks per unit of time is found upon substitution of Eq. (2.3) into Eq. (2.2), and after some algebraic operations.

$$
\begin{aligned}
& E[n(\xi, t)]=\frac{1}{4 \sqrt{2} \pi M_{\ddot{x} \ddot{x}}} \int_{\xi}^{\infty}\left[\frac{2}{\sqrt{\pi}} \sqrt{M} \exp \left(-\frac{M_{x x} x^{2}}{2|M|}\right)\right. \\
& \left.+\frac{\sqrt{2} M_{x} \ddot{x}}{\sqrt{M_{\ddot{x} \ddot{x}}}} x\left\{1+\operatorname{erf}\left(\frac{M_{x \ddot{x}} x}{2|M| M_{\ddot{x} \ddot{x}}}\right)\right\} \exp \left(\frac{M_{x \ddot{x}}^{2}-M_{x x} M_{\ddot{x} \ddot{x}}}{2|M| M_{\ddot{x} \ddot{x}}} x^{2}\right)\right] d x
\end{aligned}
$$

where

$$
\operatorname{erf}(x)=\frac{2}{\sqrt{\pi}} \int_{0}^{x} e^{-y^{2}} d y
$$

The expected total number of peaks above $\xi$ during the interval $\left(0, t_{d}\right)$ is then

$$
E\left[N\left(\xi, t_{d}\right)\right]=\int_{0}^{t_{d}} E[n(\xi, t)] d t
$$

The absolute maximum value, therefore, can be found by letting the lefthand side of Eq. (2.6) equal 1.0 or 0.5, each of which gives the lower and the upper bound, respectively.

$$
\begin{array}{ll}
\text { for the lower bound: } & E\left[N\left(\xi_{\max }^{L}, t_{d}\right)\right]=1.0 \\
\text { for the upper bound: } & E\left[N\left(\xi_{\max }^{U}, t_{d}\right)\right]=0.5
\end{array}
$$

On the other hand, the probability density of peaks at time $t$ is given, based on the heuristic assumption by Huston and Skopinski ${ }^{17)}$, as 


$$
p(\xi, t)=-\frac{1}{E\left[\left(n_{T}(t)\right]\right.} \cdot \frac{\partial}{\partial \xi} E[n(\xi, t)]
$$

where the notation $E\left[n_{T}(t)\right]$ means the expected total number of peaks per unit of time, regardless of their magnitude, and is obtained from

$$
E\left[n_{r}(t)\right]=\int_{-\infty}^{\infty} d x \int_{-\infty}^{0} \ddot{x}[p(x, \dot{x}, \ddot{x} ; t)] d \ddot{x}=0
$$

Substitution of Eqs. (2.4) and (2.9) into Eq. (2.8) yields

$$
\begin{aligned}
& p(\xi, t)=\frac{1}{2 \sqrt{2}} \cdot \frac{M_{\ddot{x}} \ddot{x} M_{x x}-M_{\ddot{x}}^{2}}{\sqrt{M_{x x} M_{\ddot{x}} \ddot{x}|M|}}\left[\frac{2}{\sqrt{\pi}} \sqrt{\bar{M}} \exp \left(-\frac{M_{x x}}{2|M|} \xi^{2}\right)\right. \\
& \left.+\frac{\sqrt{2} M_{x \ddot{x}}}{\sqrt{M_{\ddot{x}} \ddot{x}}} \xi\left\{1+\operatorname{erf}\left(\frac{M_{x \ddot{x}}}{2|M| M_{\ddot{x} \ddot{x}}} \xi\right)\right\} \exp \left(\frac{M_{x \ddot{x}}^{2}-M_{x x} M_{\ddot{x} \ddot{x}}}{2|M| M_{\ddot{x} \ddot{x}}} \xi^{2}\right)\right]
\end{aligned}
$$

\section{SIMULATION OF EARTHQUAKE MOTION}

Simulation of earthquake motion in a stochastic sense means an approximation of its spectral composition in frequency domain on the basis of strong earthquake records ${ }^{18}$ ) or on the geophysical investigation. Such a procedure has been used by many researcher, assuming a filtered Guassian stationary white noise

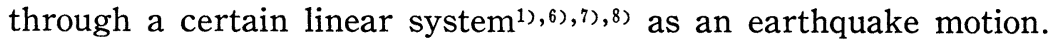

The authors also followed this semi-experimental way of thinking in considering earthquake acceleration, and it is simulated by the velocity response of a linear filter ${ }^{5}$

$$
\ddot{x}_{g}+2 \mu_{g} \dot{x}_{g}+\left(\omega_{g}{ }^{2}+\mu_{g}{ }^{2}\right) x_{g}=\ddot{x}_{b}
$$

where the notations $\omega_{g}$ and $\mu_{g}$ represent, respectively, the predominant frequency and the damping effect of the foundation concerned. Then, applying the inputoutput relationship in stochastic process to the above system, the power spectral density of the synthetic earthquake motion is

$$
\begin{aligned}
& S_{\dot{x}_{g}}(\omega)=\left|H_{\dot{x}_{g}}(i \omega)\right|^{2} D \\
& S_{\dot{x}_{g}}(\omega)=\frac{\omega^{2}}{\left(\omega_{g}{ }^{2}+u_{g}{ }^{2}-\omega^{2}\right)^{2}+4 u_{g}{ }^{2} \omega^{2}} D
\end{aligned}
$$

with the intensity $D$ at the base rock as a parameter.

Most of aarthquake acceleration records, as are shown by the dotted line in Fig. 1, have a broad-band power spectral density of one peak, or that of several peaks where a few predominant frequencies can be observed. Hence, the foundation layer of an idealized parallel system of one-degree-of-freedom systems as in Fig. 2, was assumed. This results in the following input-output relationship in frequency domain.

$$
S_{\dot{x}_{g}}(\omega)=\left|\sum_{i=1}^{m} \alpha_{i} H_{\dot{x}_{g_{i}}}(\underline{i} \omega)\right|^{2} D
$$

where the coefficients $\alpha_{i}$ determines the magnitude among peaks. In this paper, the following simplified expression of Eq. (3.4) was adopted. 

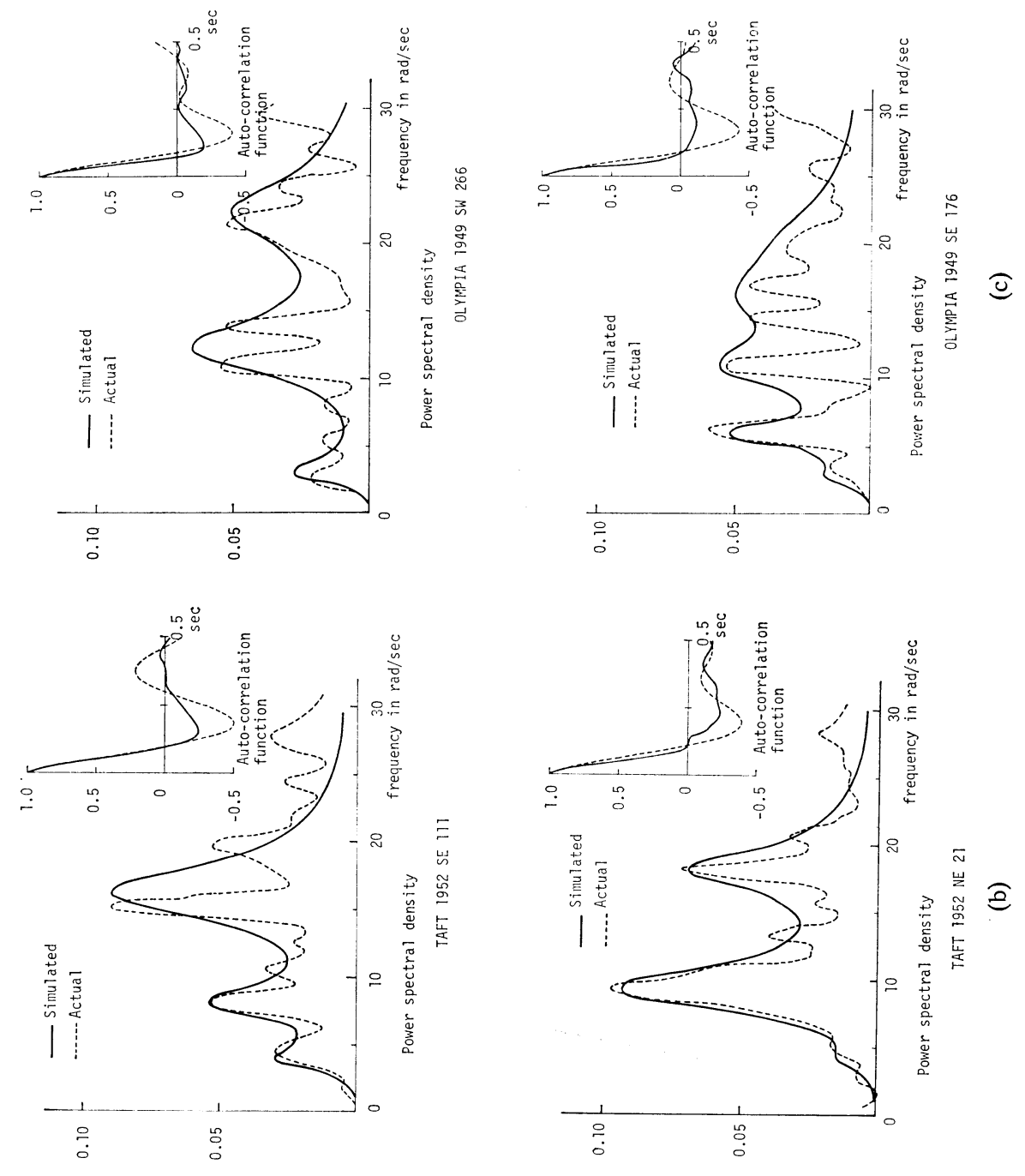

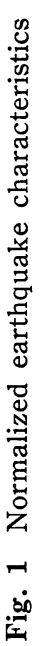
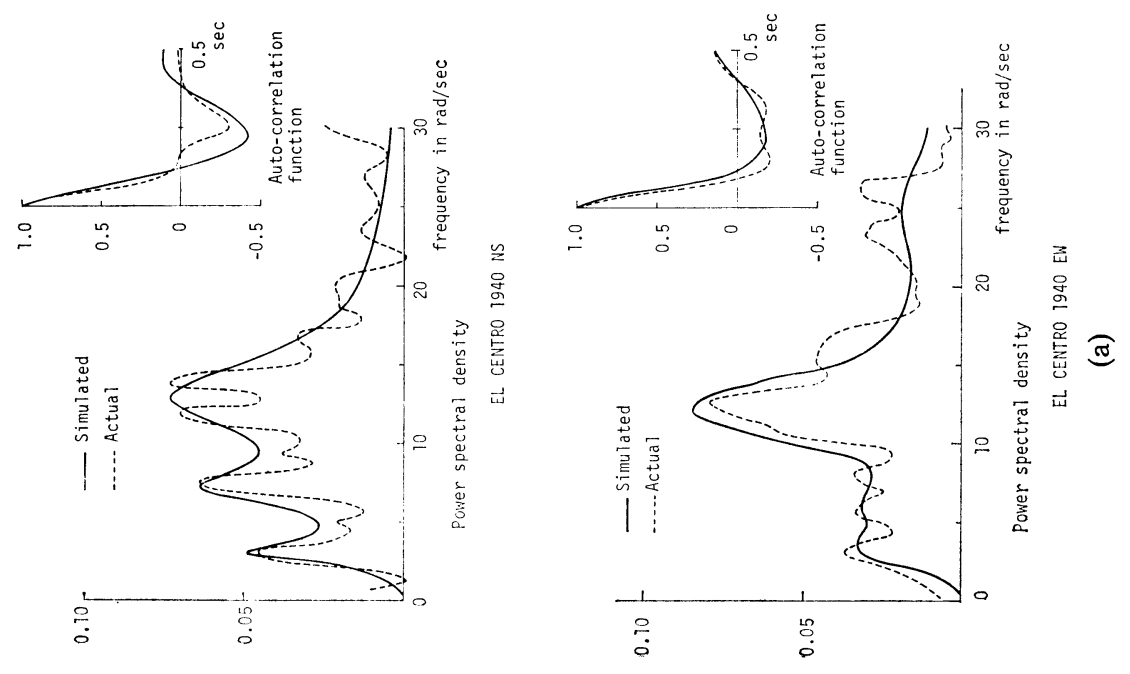


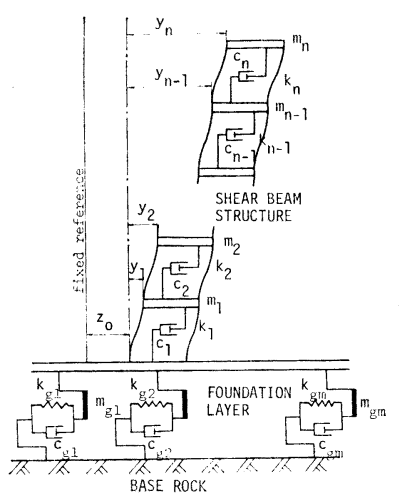

Fig. 2 Structural system

$$
S_{\dot{x}_{g}}(\omega)=\sum_{i=1}^{m} \alpha_{i}\left|H_{\dot{x}_{g_{i}}}(i \omega)\right|^{2} D
$$

This function presents a tendency closely approximate to the results of several strong earthquake records: i.e., by adjusting parameters involved, the prominent peak or peaks of the above function can be confined within the frequency range, for instance, $0 \sim 60 \mathrm{rad} / \mathrm{sec}$, and its amplitude can be made negligibly small near the zero frequency range, and diminished in an asymptotic manner at the higher range more than $60 \mathrm{rad} / \mathrm{sec}$. As fundamental information concerning the value of parameters, Figs. 3, 4 are shown, where the number of superpositions was taken up to 2 . Several illustrations are presented by the solid line in Fig. 1. Their conformity in shape to actual power spectral densities could be attained by choosing parameters in Eq. (3.5) as in Table 1 through man-machine communication (by FACOM 230-10).

As for the auto-correlation functions of the above synthetic earthquake motions, they are found as the Fourier transform of Eq. (3.5) as

$$
R_{\dot{x}_{g}}(\tau)=\sum_{i=1}^{m} \frac{\alpha_{i}}{4 \mu_{g_{i}} \omega_{g_{i}}} \exp \left(-\mu_{g_{i}}|\tau|\right)\left(\omega_{g_{i}} \cos \omega_{g_{i}} \tau-\mu_{g_{i}} \sin \omega_{g_{i}}|\tau|\right) D
$$
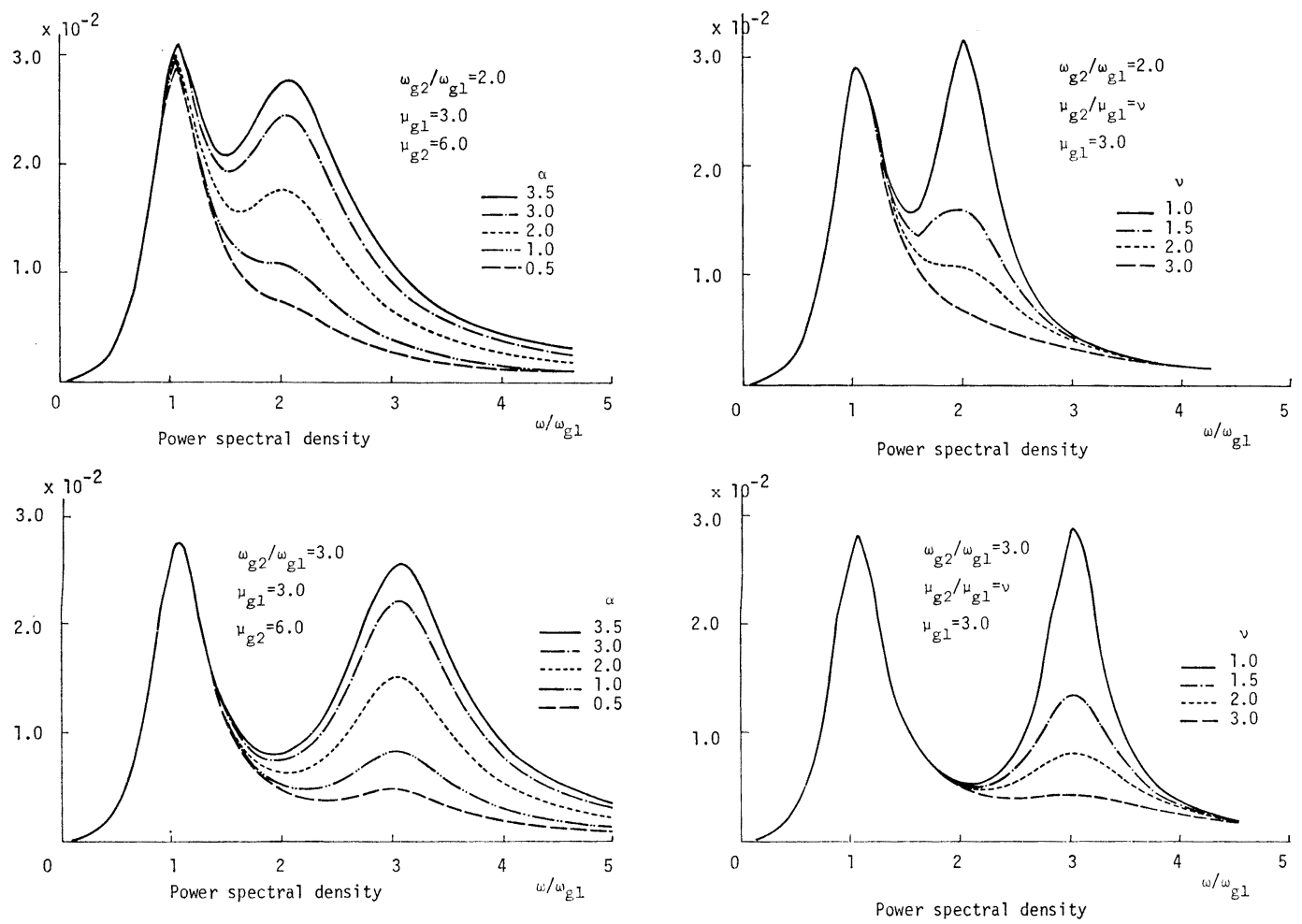

Fig. 3 Earthquake simulation effect of $\alpha$

Fig. 4 Earthquake simulation effect of $\mu$ 
Comparison between these auto-correlation functions and those of strong earthquake records is also made in Fig. 1.

\section{MAXIMUM ESTIMATION OF EARTHQUAKE ACCELERATION}

When the earthquake motions are simulated by the stochastic process, their expected mean value and moments can easily be found. However, their maxima are more significant from the aseismic structural engineering point of view. For this investigation, the theory derived in section II is certainly applicable. The necessary statistical quantities such as those included in Eq. (2.4) or (2.10) can be obtained from Eq. (3.5) and the stationarity of the concerned process as follows:

$$
\begin{aligned}
& \sigma_{F}^{2}=\frac{1}{2 \pi} \sum_{i=1}^{m} \int_{0}^{\infty}\left|H_{\dot{x}_{g_{i}}}(i \omega)\right|^{2} D d \omega \\
& \sigma_{F}^{2}=\frac{1}{2 \pi} \sum_{i=1}^{m} \int_{-\infty}^{\infty}\left|H_{\dot{x}_{g_{i}}}(i \omega)\right|^{2} D d \omega \\
& \sigma_{\dot{F}}^{2}=\frac{1}{2 \mu} \sum_{i=1}^{m} \int_{0}^{\infty} \omega^{2}\left|H_{\dot{x}_{g_{i}}}(\underline{i} \omega)\right|^{2} D d \omega \\
& \sigma_{\ddot{F}}^{2}=\frac{1}{2 \pi} \sum_{i=1}^{m} \int_{0}^{\infty} \omega^{4}\left|H_{\dot{x}_{i}}(\underline{i} \omega)\right|^{2} D d \omega \\
& \sigma_{F \dot{F}}^{2}=0 \\
& \sigma_{\dot{F} \ddot{F}}^{2}=0 \\
& \sigma_{F \ddot{F}}^{2}=-\sigma_{\dot{F}}^{2}
\end{aligned}
$$

in the case of one-sided power spectral density ${ }^{19}$

in the case of two-sided power spectral density

The actual calculations were carried out with an integral range of $0 \sim 60 \mathrm{rad} / \mathrm{sec}$. This truncation is efficacious as is proved by comparing the results by numerical integration of Eq. (4.1.a) and by residue integration of Eq. (4.1.b).

Substitution of Eq. (4.1) through Eq. (4.6) into Eq. (2.4) and Eq. (2.10) yields the expected number of peaks of the synthetic earthquake motion above the specified level $\xi$ and its probability density of peaks, which are shown in Figs. 5 and 6, respectively. Fig. 5 indicates that the maximum acceleration of earthquake motion is about 3.0 3.5 times of its standard deviation, regardless of the shape of its power spectral density, but affected by its duration as shown in Fig. 7. Furthermore, this figure predicts the following approximate relationship between the ratio of maximum to standard deviation of earthquake acceleration and its duration. 


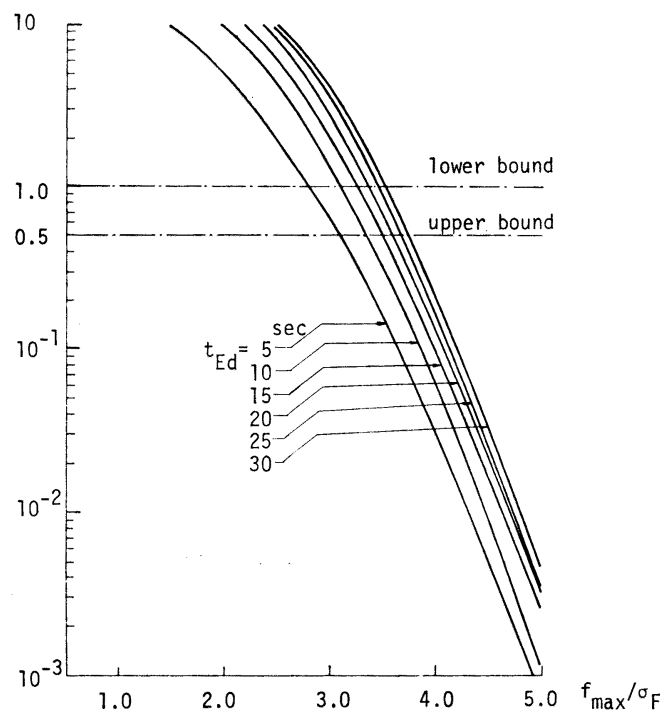

Fig. 5 Peak distribution of earthquake motion

for the lower bound

$$
\alpha_{f}^{L}=0.91 \log _{10} t_{E a}+2.16
$$

for the upper bound

$$
\alpha_{f}^{U}=0.82 \log _{10} t_{E d}+2.53
$$

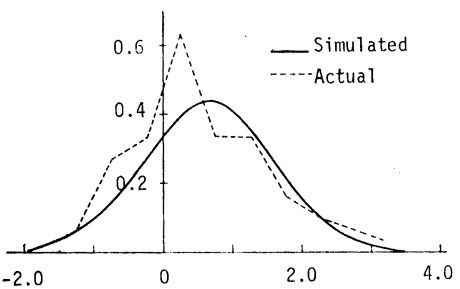

EL. CENTRO $1940 \mathrm{NS}$
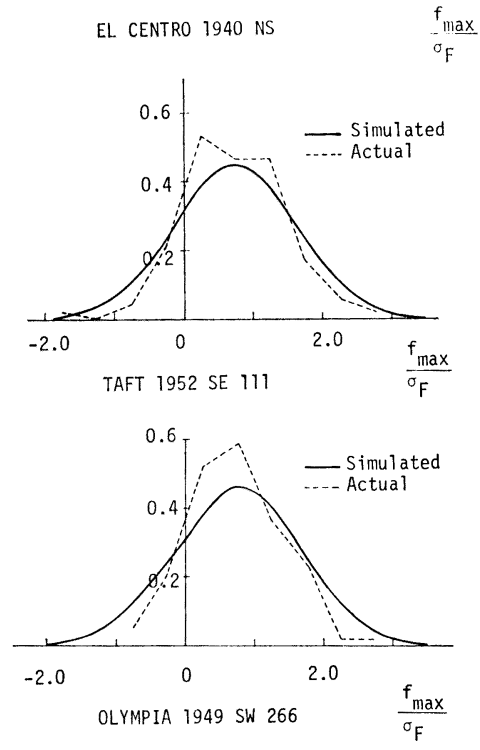

Fig. 6 Probability density of peaks

These formulae have a narrow-band estimation of the maximum. Their application to strong earthquake records, as indicated in Table 2, gives a good coinci-

\begin{tabular}{|c|c|c|c|c|c|c|c|c|c|c|c|c|}
\hline & $t_{E d}$ & $f_{\max }^{\mathrm{ob}}$ & $f_{\text {min }}^{o b}$ & $f$ & $\mathrm{u}_{\mathrm{f}}^{\mathrm{L}}$ & $f_{L, \max }^{\text {st }}$ & $\begin{array}{r}U \\
\times \quad f\end{array}$ & $f_{U, \max }^{S t}$ & (1) & (2) & (3) & (4) \\
\hline EL CENTRO 1940 NS & 10.3 & 298 & -318 & 94.3 & 3.11 & 293 & 3.38 & 319 & 1.02 & 1.08 & 0.93 & 1.00 \\
\hline EL CENTRO $1940 \mathrm{EW}$ & 9.0 & 230 & -160 & 70.2 & 3.49 & 245 & 3.73 & 261 & 0.94 & 0.65 & 0.88 & 0.61 \\
\hline TAFT 1952 SE 111 & 17.0 & 180 & -149 & 57.0 & 3.28 & 187 & 3.53 & 201 & 0.96 & 0.80 & 0.90 & 0.74 \\
\hline TAFT 1952 NE 21 & 16.0 & 170 & -182 & 56.6 & 3.26 & 184 & 3.51 & 199 & 0.92 & 0.99 & .85 & 0.91 \\
\hline OLYMPIA 1949 SE 176 & 19.0 & 160 & -193 & 69.2 & 3.32 & 230 & 3.58 & 247 & 70 & 0.84 & 0.65 & 0.78 \\
\hline OLYMPIA 1949 SW 266 & 19.0 & 307 & -193 & 84.4 & 3.32 & 281 & 3.58 & 302 & 1.09 & 0.69 & 1.02 & 0.64 \\
\hline
\end{tabular}

Table 2 Estimation of the absolute maximum acceleration of earthquakes

$$
\begin{aligned}
& (1)=f_{\max }^{o b} / f_{L, \max }^{s t} \quad(2)=-f_{\min }^{o b} / f_{L, \max }^{s t} \quad(3)=f_{\max }^{o b} / f_{U, \max }^{s t} \quad(4)=-f_{\min }^{o b} / f_{U, \max }^{s t} \\
& t_{E d} \text { : duration of earthquake record used for statistical analysis } \\
& f_{\max }^{o b} \text { : observed maximum acceleration } \\
& f_{\text {min }}^{o b} \text { : observed minimum acceleration } \\
& \sigma_{f} \text { : standard deviation of peaks of earthquake records } \\
& \alpha_{f} \text { : lower bound ratio of maximum to standard deviation, Eq. (4.7.a) } \\
& u_{f} \text { : upper bound ratio of maximum to standard deviation, Eq. (4.7.b) } \\
& f_{L \text {, max }}^{\text {st }} \text { : lower bound of the statistical maximum acceleration } \\
& f_{U, \max }^{\text {st }} \text { : upper bound of the statistical maximum acceleration }
\end{aligned}
$$




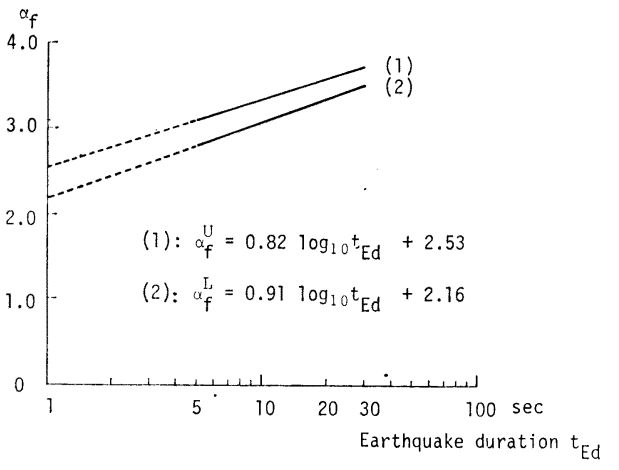

Fig. 7 Ratio of earthquake maximum acceleration to its standard deviation dence of actually observed maxima or minima. In Fig. 6 are also presented the peak distribution directly obtained from strong earthquake records for comparison. Each assumed stochastic process well represents the significant range of peak distribution-the portion which is more than 1.5 times the standard deviation, and it is concluded that the above good estimates of the maxima or minima of earthquake accelerations are due to this fact.

\section{RESPONSE OF A ONE-DEGREE- OF-FREEDOM SYSTEM}

The input-output relationship of a one-degree-of-freedom system in frequency domain, when restricted within an elastic limit, is expressed by

$$
S_{R}(\omega)=\left|{ }_{a}^{d} H(\underline{i} \omega)\right|^{2} S_{F}(\omega)
$$

where $S_{F}$ and $S_{R}$ are the power spectral densities of excitation and response, respectively. ${ }_{a}^{d} H(\omega)$ is the transfer function with regard to displacement response of one-degree-of-freedom system to acceleration input; i.e.,

$$
{ }_{a}^{a} H(\underline{i} \omega)=\frac{-1}{\left(\omega_{0}^{2}-\omega^{2}\right)+\underline{i} 2 \zeta \omega_{0} \omega}
$$

where $\omega_{0}$ and $\zeta$ are the undamped natural frequency and the damping factor, respectively. When the system is subjected to the synthetic earthquake motion of Eq. (3.5), its two-sided response power spectral density is thus obtained as

$$
S_{R}(\omega)=\sum_{i=1}^{m} \alpha_{i}|H(\underline{i} \omega)|^{2}\left|H_{\dot{x}_{i}}(i \omega)\right|^{2} D / 2
$$

The corresponding variance, as the inverse Fourier transform of this, is

$$
\sigma_{d}^{2}(t)=\frac{1}{2 \pi} \int_{-\infty}^{\infty} S_{R}(\omega) d \omega
$$

Integration of Eq. (5.4) upon substitution of Eq. (5.3) is carried out by the method of residue theory. Such integral formulae applicable here are given in reference (20) in the following form:

$$
I=\int_{-\infty}^{\infty}|H(\underline{i} \omega)|^{2} d \omega
$$

where

$$
H(\underline{i} \omega)=\frac{-\underline{i} \omega^{3} B_{3}-\omega^{2} B_{2}+\underline{i} \omega B_{i}+B_{0}}{\omega^{4} A_{4}-\underline{i} \omega^{3} A_{3}-\omega^{2} A_{2}+\underline{i} \omega A_{i}+A_{0}}
$$

and the result is 


$$
\begin{aligned}
I= & \pi\left[\left(B_{0}^{2} / A_{0}\right)\left(A_{2} A_{3}-A_{1} A_{4}\right)+A_{3}\left(B_{1}{ }^{2}-2 B_{0} B_{2}\right)+A_{1}\left(B_{2}{ }^{2}-2 B_{1} B_{3}\right)\right. \\
& \left.+\left(B_{3}{ }^{2} / A_{4}\right)\left(A_{1} A_{2}-A_{0} A_{3}\right)\right] /\left[A_{1}\left(A_{2} A_{3}-A_{1} A_{4}\right)-A_{0} A_{3}{ }^{2}\right]
\end{aligned}
$$

This kind of residue integrals were originally formulated by $\mathrm{H}$. M. James and others ${ }^{21)}$. Then, the displacement response variance is

$$
\sigma_{d}^{2}=\frac{1}{2 \pi} \sum_{i=1}^{m} \frac{I_{i}}{2}
$$

where $I_{i}$ is the integrated value by the use of Eq. (5.7) with the following coefficients:

$$
\begin{array}{ll}
A_{0 i}=\omega_{0}^{2}\left(\omega_{g_{i}}^{2}+\mu_{g_{i}}^{2}\right) & B_{0 i}=0 \\
A_{1 i}=2 \omega_{0}\left\{\zeta\left(\omega_{g_{i}}^{2}+\mu_{g_{i}}^{2}\right)+\mu_{g_{i}} \omega_{0}\right\} & B_{1 i}=-1 \\
A_{2 i}=\omega_{0}^{2}+\omega_{g_{i}}^{2}+\mu_{g_{i}}^{2}+4 \zeta \omega_{0} \mu_{g_{i}} & B_{2 i}=0 \\
A_{3 i}=2\left(\zeta \omega_{0}+\mu_{g_{i}}\right) & B_{3 i}=0 \\
A_{4 i}=1 &
\end{array}
$$

The velocity response variance is likewise

$$
\sigma_{v}^{2}=\frac{1}{2 \pi} \sum_{i=1}^{m} \int_{-\infty}^{\infty} \omega^{2}\left|{ }_{a}^{d} H(\underline{i} \omega)\right|^{2} \alpha_{i}\left|H_{\dot{x}_{g_{i}}}(\underline{i} \omega)\right|^{2} D / 2 \cdot d \omega
$$

This integration is also carried out by Eq. (5.7) with the same coefficients of $A_{j i}(j=1,4)$ as in Eq. $(5.9)$ and with $B_{j i}(j=1,4)$ of

$$
\left.\begin{array}{l}
B_{0 i}=0 \\
B_{1 i}=0 \\
B_{2 i}=-1 \\
B_{3 i}=0
\end{array}\right\}
$$

As for the absolute acceleration response variance, using the transfer function of

$$
\sigma_{a}^{2}=\frac{1}{2 \pi} \sum_{i=1}^{m} \int_{-\infty}^{\infty}\left|{ }_{a}^{d} H(\underline{i} \omega)\right|^{2} \alpha_{i}\left|H_{\dot{x}_{g_{i}}}(\underline{i} \omega)\right|^{2} D / 2 \cdot d \omega
$$

instead of Eq. (5.2), it is found to be

$$
{ }_{a}^{d} H(i \omega)=\frac{\omega_{0}^{2}+i 2 \zeta \omega_{0} \omega}{\omega_{0}^{2}-\omega^{2}+\underline{i} 2 \zeta \omega_{0} \omega}
$$

The integrated value is obtained by Eq. (5.7) with the same coefficients of $A_{j i}$ $(j=1,4)$ in Eq. $(5.9)$ and the following $B_{j i}$ :

$$
\left.\begin{array}{l}
B_{0 i}=0 \\
B_{1 i}=\omega_{0}^{2}
\end{array}\right\}
$$




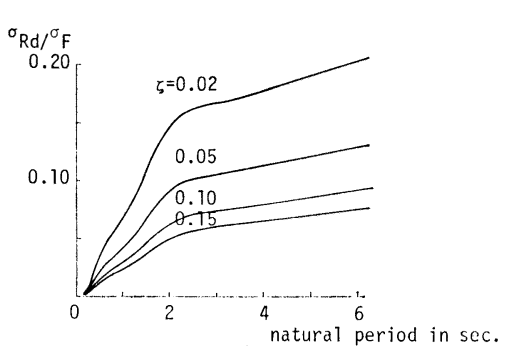

Fig. 8 Deformation amplification spectrum by stochastic analysis

$$
\begin{aligned}
& B_{2 i}=2 \zeta \omega_{0} \\
& B_{3 i}=0
\end{aligned}
$$

Figs. 8, 9, 10, which were obtained through averaging the response to the synthetic earthquake motions in section III, represent the response amplification factors in a statistical sense with regard to deformation, velocity and absolute acceleration, respectively. Their general features closely approximate those to actual strong earthquakes ${ }^{22}$ ) in the range less than about $3 \mathrm{rad} / \mathrm{sec}$. The precise maximum spectra, however, may be the different ones from the above figures in the range more than that, especially so the deformation spectrum as is investigated below.

\section{MAXIMUM RESPONSE OF A ONE-DEGREE-OF-FREEDOM SYSTEM}

Maximum response of a lightly damped system is more easily calculated by applying the Rayleigh distribution rather than by Eq. (2.10) for its peak distribution of response. The former distribution is a special case of the latter with a random index $\varepsilon=0^{23)}$. In this case the number of peaks is supposedly between the number of zero-crossing with upward (or downward) and its twice. Hence, the following equations with regard to the lower and the upper bounds of the number of peaks above $\xi$.

for the lower bound

$$
E\left[N^{L}\left(\xi, t_{d}\right)\right]=\int_{0}^{t_{\mathrm{Rd}}} E\left[n\left(0_{+}\right)\right] \int_{\xi}^{\infty} p(x) d x d t
$$

for the upper bound

$$
E\left[N^{U}\left(\xi, t_{d}\right)\right]=\int_{0}^{t_{\mathrm{Rd}}} 2 E\left[n\left(0_{+}\right)\right] \int_{\xi}^{\infty} p(x) d x d t
$$

where the number of zero-crossing with upward is obtained by Eq. (2.9) as

$$
E\left[n\left(0_{+}\right)\right]=\frac{1}{2 \pi} \cdot \frac{\boldsymbol{\sigma}_{v}}{\boldsymbol{\sigma}_{d}}
$$


and the Rayleigh distribution function is expressed by

$$
p(x)=\frac{x}{\sigma_{d}^{2}} \exp \left(-\frac{x^{2}}{2 \sigma_{d}^{2}}\right)
$$

After integration of Eq. (6.1) over the variable $x$ from $\xi$ to infinite, it becomes as

for the lower bound

$$
E\left[N^{L}\left(\xi, t_{d}\right)\right]=\int_{0}^{t_{\mathrm{Rd}}} E\left[n\left(0_{+}\right)\right] \exp \left(-\frac{\xi^{2}}{2 \sigma_{d}^{2}}\right) d t
$$

for the upper bound

$$
E\left[N^{U}\left(\xi, t_{d}\right)\right]=\int_{0}^{t_{\mathrm{Rd}}} 2 E\left[n\left(0_{+}\right)\right] \exp \left(-\frac{\xi^{2}}{2 \sigma_{d}^{2}}\right) d t
$$

Maximum response during the motion can thus be found by letting the righthand side of Eq. (6.4) equal 1, which leads the following ratio of maximum to standard deviation response:

for the lower bound

$$
\frac{d_{\max }^{L}}{\sigma_{d}}=\sqrt{2 \log _{e}\left(E\left[n\left(0_{+}\right)\right] t_{\mathrm{Rd}}\right)}
$$

for the upper bound

$$
\frac{d_{\max }^{U}}{\sigma_{d}}=\sqrt{2 \log _{e}\left(2 E\left[n\left(0_{+}\right)\right] t_{\mathrm{Rd}}\right)}
$$

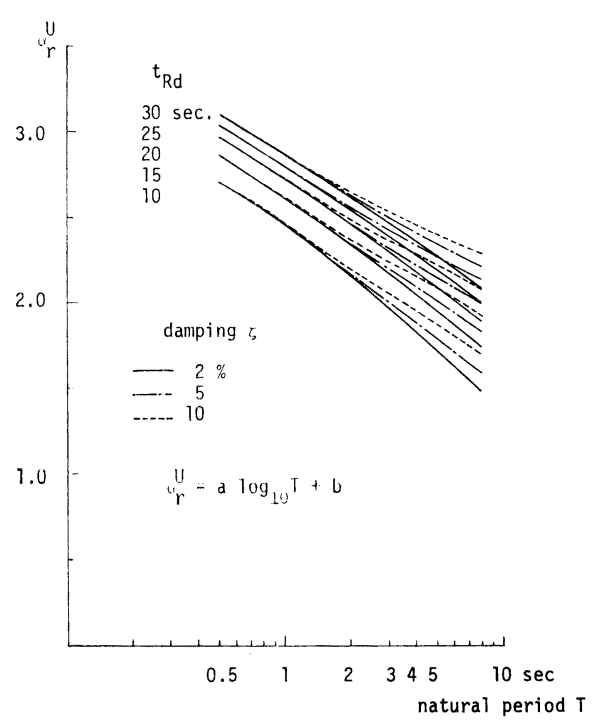

Fig. 11 Ratio of maximum response to its standard deviation (upper bound)
These ratios have almost identical tendencies regardless of the input power spectral density as are shown in Fig. 11. They very proportionally in a logarithmic as the natural period of the system. Hence, the following formulae are propose for them:

for the lower bound

$$
\alpha_{r}^{L}=a^{L} \log _{10} T+b^{L}
$$

for the upper bound

$$
\alpha_{r}{ }^{U}=a^{U} \log _{10} T+b^{U}
$$

where the coefficients $a$ and $b$, which are the function of the response duration of the system and its damping effect, are listed in Table 3.

Substitution of Eqs. (4.7) and (6.6) into the result by spectral analysis of a given system yields its maximum deformation 
Table 3 Ratio of response maximum to its standard deviation

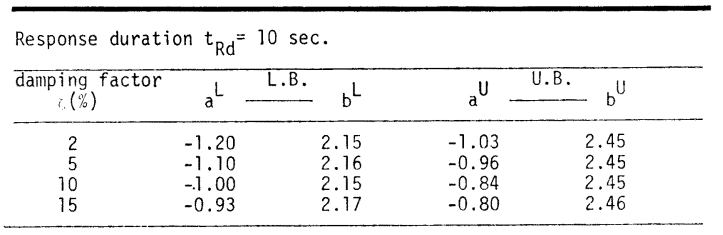

\begin{tabular}{|c|c|c|c|c|}
\hline $\begin{array}{c}\text { damping factor } \\
\qquad(\%)\end{array}$ & $a^{L}$ & L.B. $b^{L}$ & $a^{U}$ & U.B. $b^{U}$ \\
\hline $\begin{array}{r}2 \\
5 \\
10 \\
15\end{array}$ & $\begin{array}{l}-1.07 \\
-1.00 \\
-0.88 \\
-0.82\end{array}$ & $\begin{array}{l}2.33 \\
2.33 \\
2.34 \\
2.35\end{array}$ & $\begin{array}{l}-0.94 \\
-0.86 \\
-0.77 \\
-0.73\end{array}$ & $\begin{array}{l}2.61 \\
2.60 \\
2.62 \\
2.63\end{array}$ \\
\hline
\end{tabular}

Response duration $t_{R d}=20 \mathrm{sec}$.

\begin{tabular}{|c|c|c|c|c|}
\hline$\underset{\zeta(\%)}{\operatorname{damping}}$ factor & $a^{L}$ & $\stackrel{\text { L.B. }}{ } b^{L}$ & $a^{U}$ & U.B. $b^{U}$ \\
\hline $\begin{array}{r}2 \\
5 \\
10 \\
15\end{array}$ & $\begin{array}{l}-0.95 \\
-0.91 \\
-0.83 \\
-0.77\end{array}$ & $\begin{array}{l}2.45 \\
2.44 \\
2.46 \\
2.48\end{array}$ & $\begin{array}{l}-0.90 \\
-0.82 \\
-0.74 \\
-0.69\end{array}$ & $\begin{array}{l}2.72 \\
2.71 \\
2.74 \\
2.74\end{array}$ \\
\hline
\end{tabular}

Response duration $t_{R d}=25 \mathrm{sec}$.

\begin{tabular}{cccccc}
\hline $\begin{array}{c}\text { daming factor } \\
C(\%)\end{array}$ & $a^{L}$ & L.B. & $b^{L}$ & $a^{U}$ & U.B. \\
\cline { 5 - 6 } & $b^{U}$ \\
\hline 2 & -0.96 & 2.55 & -0.87 & 2.80 \\
5 & -0.84 & 2.54 & -0.78 & 2.80 \\
10 & -0.79 & 2.55 & -0.72 & 2.81 \\
15 & -0.73 & 2.56 & -0.67 & 2.83 \\
\hline
\end{tabular}

Response duration $t_{\mathrm{Rd}}=30 \mathrm{sec}$.

\begin{tabular}{|c|c|c|c|c|}
\hline$\underset{\tau(c)}{\text { damping factor }}$ & $a^{L}$ & L.B. $b^{L}$ & $a^{\text {U }}$ & U.B. \\
\hline $\begin{array}{r}2 \\
5 \\
10 \\
15\end{array}$ & $\begin{array}{l}-0.94 \\
-0.85 \\
-0.78 \\
-0.71\end{array}$ & $\begin{array}{l}2.61 \\
2.61 \\
2.63 \\
2.63\end{array}$ & $\begin{array}{l}-0.83 \\
-0.75 \\
-0.69 \\
-0.67\end{array}$ & $\begin{array}{l}2.86 \\
2.87 \\
2.89 \\
2.89\end{array}$ \\
\hline
\end{tabular}

response as the function of input maximum acceleration.

$$
d_{\max }=\left(\frac{\alpha_{r}}{\alpha_{f}}\right)\left(\frac{\sigma_{R}}{\sigma_{F}}\right) f_{\max }
$$

where $\left(\sigma_{R} / \sigma_{F}\right)$ is the response amplification factor in Fig. 8. In Fig. 12 the deformation spectrum obtained herein is compared with the one proposed by Y. Nakao and N. Sasaki ${ }^{15)}$, and the one by the Research Institute of Construction Ministry of Japan ${ }^{24)}$, where the maximum input acceleration was set to equal 200 gal. The result in this paper shows a flat spectrum against others in the range

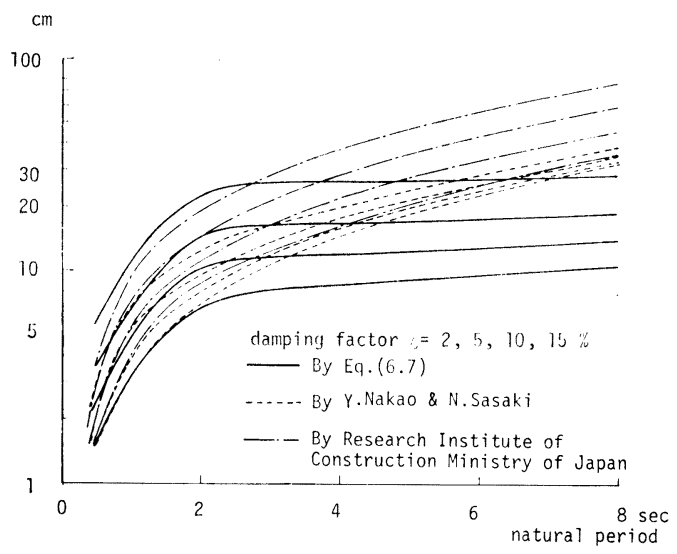

Fig. 12 Deformation spectra

Maximum acceleration of earthquake was set to eqaul $200 \mathrm{gal}$ 
more than $2.5 \mathrm{sec}$ of the natural period. This tendency is more rational from the fact that earthquake motions appear directly in the system response when the system has a long vibration period one.

\section{MAXIMUM RESPONSE OF A MULTI-DEGREE-OF-FREEDOM SYSTEM WITH APPLICATION TO A MULTI-STORY BUILDING}

Response analysis of lightly damped structure with multi-degree-of-freedom is usually carried out by the modal method, with the displacement expressed as

$$
y_{k}(t)=\sum_{i=1}^{n} q_{i}(t) \phi_{k}^{(i)}
$$

where $n$ is the number of degree-of-freedom of a concerned structure and $\phi_{k}(i)$ is the $i$-th mode shape function of its $k$-section. The time function $q_{i}(t)$ is governed by the following equation.

$$
\ddot{q}_{i}+2 \zeta_{i} \omega_{0 i} \dot{q}+\omega_{0 i}^{2} q_{i}=-\beta_{i} f
$$

with notations $\omega_{0 i}$ and $\zeta_{i}$ being the corresponding undamped natural frequency and damping factor, respectively. The participation factor $\beta_{i}$ shares the $i$-th modal contribution to the structural response and it is obtained as

$$
\beta_{i}=\frac{\sum_{k=1}^{n} m_{k} \phi_{k}^{(i)}}{\sum_{k=1}^{n} m_{k}\left\{\phi_{k}^{(i)}\right\}^{2}}
$$

where $m_{k}$ is the concentrated mass at $k$-section of the structure. However, in the case of random excitation input such as earthquake motions, the above superposition of Eq. (7.1) must be replaced by the following through spectral analysis.

$$
\begin{aligned}
& \sigma_{y_{k}}^{2}(t)=E\left[y_{k}{ }^{2}(t)\right] \\
& \sigma_{y_{k}}^{2}(t)=\left[\sum_{i=1}^{n} J_{i i}\left\{\beta_{i} \phi_{k}^{(i)}\right\}^{2}+2 \sum_{i<j}^{n} J_{i j} \phi_{k}^{(i)} \phi_{k}^{(j)}\right] \sigma_{F}{ }^{2}
\end{aligned}
$$

where

$$
\begin{gathered}
J_{i i}=\left[\frac{1}{2 \pi} \int_{-\infty}^{\infty}\left|{ }_{a}^{d} H_{i}(\underline{i} \omega)\right|^{2} S_{F}(\omega) d \omega\right] /\left[\frac{1}{2 \pi} \int_{-\infty}^{\infty} S_{F}(\omega) d \omega\right] \\
J_{i j}=\left[\frac{1}{2 \pi} \int_{-\infty}^{\infty} \mathcal{R}\left({ }_{a}^{d} H_{i}(\underline{i} \omega)_{a}^{d} H_{i} *(\underline{i} \omega)\right) S_{F}(\omega) d \omega\right] /\left[\frac{1}{2 \pi} \int_{-\infty}^{\infty} S_{F}(\omega) d \omega\right] \\
\sigma_{F}^{2}=\frac{1}{2 \pi} \int_{-\infty}^{\infty} S_{F}(\omega) d \omega \\
{ }_{a}^{d} H(\underline{i} \omega)=\frac{-1}{\omega_{0 i}^{2}-\omega^{2}+\underline{i} 2 \zeta_{i} \omega_{0 i} \omega}
\end{gathered}
$$

In Eq. (7.5), the first part is the direct-contributions of each normal mode, and the second, the cross-contributions between them. The latter becomes significantly large in proportion to the closeness between adjacents modes and the value of 
their damping effects: i.e. ${ }^{8}$,

$$
\text { If } 1-\left(\frac{\omega_{i}}{\omega_{j}}\right)^{2} \gg 4 \beta_{j}^{2}\left\{\left(\frac{\omega_{i}}{\omega_{j}}\right)+2\right\} ; \quad \omega_{j}>\omega_{i}, \quad \beta_{j}=\beta_{i}
$$

then the cross-contribution between $i$-th and $j$-th mode is negligibly small

Moreover, according to the results from investigation of a tower and pier system of a long span suspension bridge ${ }^{8)}$, structures of good performance from the aseismic design point of view are the ones with their vibration modes sufficiently separated. Then, adopting the same consideration in the previous section, the maximum response for this case is obtained as

$$
y_{\kappa \max }=\sqrt{\sum_{i=1}^{n} \alpha_{r_{i}}^{2} \gamma_{i}{ }^{2}\left\{\beta_{i} \phi_{\kappa}{ }^{(i)}\right\}^{2}} \cdot \sigma_{F}
$$

where the notation $\alpha_{r_{i}}$ coincides with $\alpha_{r}$ in Eq. (6.7) at the natural period of the $i$-th mode of the concerned structure, and $\gamma_{i}$ is the deformation amplification factor in a stochastic sense with regard to $i$-th mode. This formula is expressed as the function of the maximum input acceleration upon substitution of Eq. (4.7).

$$
y_{\kappa \max }=\sqrt{\sum_{i=1}^{n} \alpha_{r_{i}}^{2} \gamma_{i}{ }^{2}\left\{\beta_{i} \phi_{\kappa}^{(i)}\right\}^{2}} \cdot f_{\max } / \alpha_{f}
$$

\section{Example}

A 33-story building, idealized with an equivalent 5-mass system was analyzed as an illustrative example of the above theory. Its dynamic properties are presented in Fig. 13 and Table 4. The design details are contained in reference (25).

In Table 5 are listed the computation results by the direct integration ${ }^{26)}$

\begin{tabular}{llllll}
\hline vibration modes & 1 -st & 2-nd & 3 -rd & 4 -th & 5 -th \\
\hline $\begin{array}{c}\text { natural period } \\
(\text { sec. })\end{array}$ & 4.20 & 1.73 & 1.14 & 0.87 & 0.70 \\
\hline
\end{tabular}

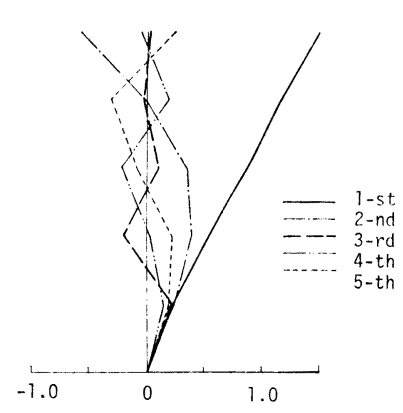

Fig. 13 Mode shapes

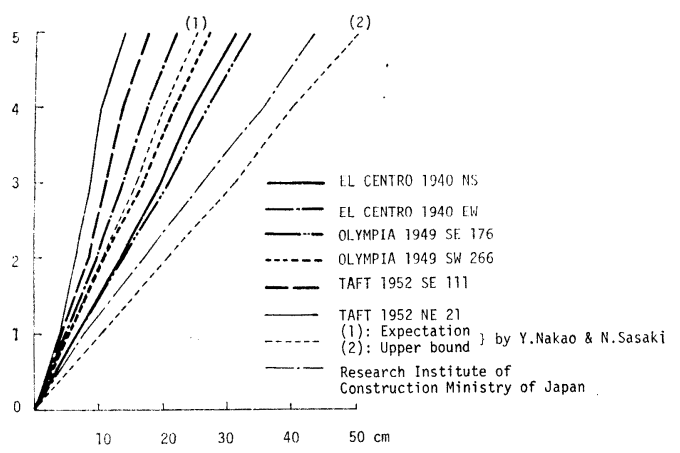

Fig. 14 Maximum deformation response

Maximum input acceleration $200 \mathrm{gal}$

Response duration $t_{\mathrm{Rd}}=25 \mathrm{sec}$

Fraction of damping $\zeta=0.05$ of each mode 
Table 5 Maximum response

\begin{tabular}{|c|c|c|c|c|c|}
\hline \multicolumn{2}{|c|}{$\begin{array}{l}\text { Direct-integrated } \\
\text { response(by } \beta \text {-method) }\end{array}$} & $\begin{array}{c}\begin{array}{c}\text { Response by } \\
\text { L.B. }\end{array} \\
5.3 \mathrm{~cm}\end{array}$ & $\begin{array}{l}\text { Eq. }(7.12)^{x} \\
\text { U.B. }\end{array}$ & \multicolumn{2}{|c|}{$\begin{array}{l}\text { Response by Y.Nakao \& N.Sasaki } \\
\text { Expectation U.B. }\end{array}$} \\
\hline $\begin{array}{l}\text { SEC. } 1 \\
\text { SEC. } \\
\text { SEC. } \\
\text { SEC. } 4 \\
\text { SEC. } 5\end{array}$ & $\begin{array}{l}4.6 \mathrm{~cm} \\
10.1 \\
17.3 \\
25.0 \\
30.8\end{array}$ & $\begin{array}{l}5.3 \mathrm{~cm} \\
10.8 \\
15.5 \\
19.8 \\
25.1\end{array}$ & $\begin{array}{r}6.5 \mathrm{~cm} \\
13.3 \\
19.3 \\
24.6 \\
31.7\end{array}$ & $\begin{array}{l}9.1 \mathrm{~cm} \\
19.2 \\
28.4 \\
36.6 \\
45.0\end{array}$ & $\begin{array}{l}18.2 \mathrm{~cm} \\
38.3 \\
56.8 \\
73.2 \\
91.9\end{array}$ \\
\hline
\end{tabular}

TAFT 1952 NE 21

\begin{tabular}{lrrrrr}
\hline $\begin{array}{l}\text { Direct-integrated } \\
\text { response(by B-method) }\end{array}$ & $\begin{array}{c}\text { Response by } \begin{array}{c}\text { Eq. (7.12) } \\
\text { L.B. }\end{array} \\
\text { U.B. }\end{array}$ & $\begin{array}{c}\text { Response by Y.Nakao \& N.Sasaki } \\
\text { Expectation } \\
\text { U.B. }\end{array}$ \\
\hline SEC.1 & $4.1 \mathrm{~cm}$ & $3.2 \mathrm{~cm}$ & $3.6 \mathrm{~cm}$ & $4.7 \mathrm{~cm}$ & $9.4 \mathrm{~cm}$ \\
SEC.2 & 5.7 & 5.5 & 6.3 & 8.7 & 17.3 \\
SEC.3 & 8.1 & 7.5 & 8.6 & 12.1 & 24.3 \\
SEC.4 & 9.6 & 9.3 & 10.7 & 15.3 & 30.6 \\
SEC.5 & 12.3 & 12.0 & 13.7 & 19.5 & 40.0 \\
\hline
\end{tabular}

TAFT 1952 SE 111

\begin{tabular}{llcccc}
\hline $\begin{array}{l}\text { Direct-integration } \\
\text { response(by B-method) }\end{array}$ & $\begin{array}{c}\text { Response by Eq. } \\
\text { L.B. }\end{array}$ & $\begin{array}{c}\text { U.B. } \\
\text { U.B }\end{array}$ & $\begin{array}{c}\text { Response by Y.Nakao \& N.Sasaki } \\
\text { Expectation } \\
\text { U.B. }\end{array}$ \\
\hline SEC.1 & $4.7 \mathrm{~cm}$ & $3.8 \mathrm{~cm}$ & $4.3 \mathrm{~cm}$ & $5.7 \mathrm{~cm}$ & $11.4 \mathrm{~cm}$ \\
SEC.2 & 8.6 & 7.1 & 8.1 & 11.2 & 22.5 \\
SEC.3 & 11.5 & 9.5 & 11.0 & 15.5 & 31.1 \\
SEC.4 & 13.3 & 11.5 & 13.3 & 19.3 & 38.6 \\
SEC.5 & 16.0 & 15.2 & 17.5 & 25.1 & 50.1 \\
\hline
\end{tabular}

* Rosponse duration $25 \mathrm{sec}$.

of the governing equation of the concerned structure with an input excitation of actual strong earthquake records, and the results by using Eq. (7.12) and by the proposal of $\mathrm{Y}$. $\mathrm{NakaO}^{15}$ ) with an input of their respective simulated power spectral densities. Our results show a good coincidence with a narrow band estimation to the precise structural response to earthquakes. This fact guarantees Eq. (7.12) strongly for estimation of the maximum response of structures to earthquake motions. Fig. 14 presents the comparison among maximum response by our proposing formula, by $\mathrm{Y}$. $\mathrm{Nakao}^{15)}$, and by the Research Institute of Construction Ministry of Japan ${ }^{24)}$, indicating that structural response is severely affected by the input power spectral density of earthquake motion. Therefore, the precise determination of the former requires the latter of well estimated. This will be possible through analytical or experimental geophysical investigations of the construction site of a structure.

\section{CONCLUSIONS}

The following conclusions are derived based on the results of this analytical study.

1. Simulation of earthquake motion adopted in this paper proved to be valid and useful in structural response analysis. It makes possible any arbitrary shaped function for the power spectral density of earthquake motion.

2 . The ratio of maximum acceleration of earthquake motion, $f_{\max }$ to its 
standard deviation, $\sigma_{f}$, regardless of the shape of its power spectral density, can be expressed only as a function of its duration $t_{E d}$, as

$$
\begin{array}{ll}
\text { for the lower bound } & \sigma_{f}{ }^{L}=0.91 \log _{10} t_{E d}+2.16 \\
\text { for the upper bound } & \sigma_{f}{ }^{U}=0.82 \log _{10} t_{E d}+2.53
\end{array}
$$

3. The ratio of maximum response of a one-degree-of-freedom system, $R_{\max }$ to its standard deviation response, $\sigma_{R}$ can be expressed as a function of its vibration period, $T$, as

$$
\alpha_{r}=a \log _{10} T+b
$$

where the coefficients $a$ and $b$ are different according to the response duration of the system and its damping effect, and are listed in Table 3.

4. Combination of the above results (1) and (2) with the input-output relationship in frequency domain of a concerned system provides the following formula as to its maximum response.

$$
d_{\max }=\left(\frac{\alpha_{r}}{\alpha_{f}}\right)\left(\frac{\alpha_{R}}{\alpha_{F}}\right) f_{\max }
$$

This formula can readily be extended to a structure with multi-degree-of-freedom system when it has well separated vibration modes.

$$
y_{\max }=\sqrt{\sum_{i}^{n} \alpha_{r_{i}}^{2} \gamma_{i}\left\{\beta_{i} \phi^{(i)}\right\}^{2}} \cdot f_{\max } / \alpha_{f}
$$

5. Computation results of a 33-story building gave a good coincidence to the response obtained by direct integration of its governing equation as an input of strong earthquake records.

\section{BIBLIOGRAPHY}

1) H. Tajimi: Basic Theories on Aseismic Design of Structure, Report of the Institute of Industrial Science, University of Tokyo, Vol. 8, No. 4, March, 1959, pp. 165-215.

2) V. V. Bolotin: Statistical Theory of the Aseismic Design of Structures, Proc. of 2nd World Conference on Earthquake Engineering, Vol. II, 1960, pp. 1365-1373.

3) J. L. Bogdanoff, J. E. Goldberg and M. Bernard: Response of a Simple Structure to a Random Earthquake-Type Disturbance, Bull. Seismol. Soc. Amer., Vol. 51, No. 2, April, 1961, pp. 293-310.

4) Y. K. Lin: Nonstationary Excitation and Response in Linear Systems Treated as Sequences of Random Pulses, J. Acoust· Soc. Am., 38, 1965, pp. 453-460.

5) M. Shinozuka and Y. Sato: Simulation of Nonstationary Process, Journal of the Engineering Mechanics Division, ASCE, Vol. 93, No. EM1, 1967, pp. 11-40.

6) M. Amin and A. H.-S Ang: Nonstationary Stochastic Model of Earthquake Motion, Proc. ASCE, Vol. 94, No. EM2, April 1968, pp. 559-583.

7) H. Goto and H. Kameda: A Statistical Study of the Maximum Ground Motion in Strong Earthquakes, Trans. JSCE, No. 159, Nov., 1968, pp. 1-12.

8) Y. Yamada and H. Takemiya: Studies on the Response of Multi-degree-of-freedom Systems Subjected to Random Excitation with Application to the Tower and Pier Systems of Long Span Suspension Bridges, Trans. JSCE, No. 163, March, 1969, pp. 17-27.

9) H. Goto and K. Toki: Structural Response to Nonstationary Random Excitation, Proc. 
of the 4th World Conference on Earthquake Engineering, Vol. A-1, 1969, pp. 130-144.

10) Y. Yamada and H. Takemiya: Studies on the Statistical Aseismic Safety of Relatively Long Period Structures, Proc. of JSCE, No. 172, Dec., 1969, pp. 63-78.

11) J-N. Yang and M. Shinozuka: Numerical Fourier Transform in Random Vibration, Journal of Engineering Mechanics Division, ASCE, Vol. 95, No. EM3, June, 1969, pp. 731746.

12) S. O. Rice: Mathematical Analysis of Random Noise, Selected Papers on Noise and Stochastic Process, edited by N. Wax, Dover, New York, 1954.

13) D. Middleton: An Introduction to Statistical Communication Theory, McGraw-Hill, New York, 1960, pp. 426.

14) H. Sato: Response of Structure System to a Model Earthquake Motion with Two Predominant Periods, Jour. of Inst. of Industrial Science, University of Tokyo, Vol. 21, No. 11, 1969, pp. 10-13.

15) Y. Nakao and N. Sasaki: Study on Aseismic Design by Statistical Method, Mitsubishi Heavy Industries Technical Review, Vol. 6, No. 1, 1969, pp. 58-69.

16) Y. K. Lin: Probabilistic Theory of Structural Dynamics, McGraw-Hill, 1967, pp. 293309.

17) W. B. Huston and T. H. Skopinski: Probability and Frequency Characteristics of Some Flight Buffet Loads, NACA TN3733, August, 1956.

18) A. Ravara: Spectral Analysis of Seismic Actions, Proc. 3rd World Conference on Earthquake Engineering, New Zealand, Vol. III, pp. 195-204.

19) J. S. Bendat and A. G. Piersol: Measurement and Analysis of Random Data, Wiley, 1966, pp. 82.

20) S. H. Crandall and W. D. Mark: Random Vibration in Mechanical Systems, Academic Press Inc., 1963, pp. 72.

21) H. M. James, N. B. Nicholas and R. S. Phillips: Theory of Servomechanisms, M.I.T. Radiation Laboratory Series, Vol. 25, pp. 333-369, McGraw-Hill, New York, 1947.

22) G. W. Housner: Vibration of Structures Induced by Seismic Waves, Schock and Vibration Handbook by C. M. Harris \& C. E. Crede, pp. 50.12-14.

23) D. E. Cartright and M. S. Longuet-Higgins: The Statistical Distribution of the Maximum of a Random Function, Proc. of the Royal Soc. of London, No. 237, 1956, pp. 212232.

24) Japan Society of Civil Engineers: Report on the Earthquake-resistant Design of HonshuShikoku Strait Bridge, App. Vol. 2, 1967, pp. 12.

25) SERAC Committee: SERAC Report, No. 5 Part V, 1964, pp. 177-234.

26) N. M. Newmark: Computation of Dynamic Structural Response in the Range Approaching Failure, Proc. of Symposium of Earthquake and Blast Effects of Structures, 1952, pp. 114-128. 